\title{
Synthesis and characterization of two-stage curing reactive bio- based polymers
}

\author{
Jing Jing Ang, Tuck Whye Wong ", Zulhairun Abdul Karim \\ Advanced Membrane Technology Research Centre (AMTEC), Universiti Teknologi Malaysia, 81310 UTM Johor Bahru, Johor, Malaysia
}

*Corresponding author: wongtuckwhye@utm.my

\section{Article history}

Received 5 May 2018

Revised 1 June 2018

Accepted 2 July 2018

Published Online 3 December 2018

\begin{abstract}
Two-stage curing reactive bio-based polymers network namely, poly(octanediol-co-dodecanedioateco-citrate-co-itaconate) (PODCl) were successfully synthesized using 1,8-octanediol, citric acid, itaconic acid and 1,12-dodecanedioic acid. In the first curing stage, a prepolymer of PODCI was thermally crosslinked. At a later time, a second curing was completed thru photo-initiated free-radical polymerization. Here, we discovered that permanent shape of the thermally crosslinked PODCI was successfully reconfigured by application of second stage curing. The molar ratio of itaconic acid (IA) to citric acid (CA) and time of photo-curing was varied. Based on XRD analysis, the polymer crystallinity of the bio-based polymer decreased with time of photo-curing. The swelling ratio of bio-based polymer decreased from $2750 \%$ to $250 \%$ as soon as completion of second stage curing. PODCl existed as a semi-crystalline polymer with a melting transition temperature of $40.2{ }^{\circ} \mathrm{C}$. Also, PODCl exhibited excellent shape memory properties with shape recovery by nearly $100 \%$.
\end{abstract}

Keywords: Bio-based polymer, shape memory property, photo-induced free radical polymerization

(c) 2018 Penerbit UTM Press. All rights reserved

\section{INTRODUCTION}

Petroleum-based polymers are widely used in packaging and plastics manufacturing industry. Due to the environmentally unfriendly and waste disposal landfill problem, the petroleum-based polymers raised the warning sign to worldwide. Replacing the petroleum-based polymers with those derived from bio-masses may seem to be a costeffective and sustainable approach. Bio-based precursors can be derived from bio-masses, which may include but not limited to crops, aquatic plants, wood and animal waste. Consequently, bio-based polymers sound sustainable, eco-efficient, and environment-friendly whereas petroleum-based polymers are the major components of landfill due to non-biodegradable (Miao et al., 2013).

The thermally crosslinked bio-based polymer possessed irreversible crosslink within the polymer network. Due to major biobased polymer undergone thermally crosslink process, in this way they cannot be remolded or reshape (Yang et al., 2004, \& Serrano et al., 2011). However, by incorporating a second crosslink curable monomer, new crosslinks will be formed within the polymer network. Some of the polymers that use to create a new shape configuration by undergoing two different curing stages, which are thermal curing first then followed by the photoinitiated curing process. A UV curable monomer was incorporated into the polymer network, which is assisting for new shape configuration. (Meng et al., 2014, \& Yakacki et al., 2015).

Interestingly, shape-memory properties of these bio-based polymers also have been revealed. The first US patent related to shape memory polymer was registered in the year 1941, it is about synthesis and testing on a dental material that made use of methacrylic acid ester resin. This material was found able to recovers its original shape when triggered by heat (Liu et al., 2007). Shape-memory polymers (SMP) are a class of smart materials that can be fixed in a temporary shape and regain its permanent shape upon triggered by an external stimulus, typical heat (Xie, 2011). SMP was can be found in a number of applications ranged from aerospace, automotive, and most promisingly, in the medical field.
In this project, a series of two stages curing reactive polymer networks were successfully synthesized using 1,8-octanediol, citric acid, itaconic acid, and 1,12-dodecanedioic acid. Here, it was expected that the two-stage curing method can be utilized to the re-configure permanent shape of a thermally crosslinked polymer. We studied the change in structural, thermal, and shape-memory properties of the biobased polymers upon two-stage curing.

\section{EXPERIMENTAL}

\section{Materials}

1,8-octanediol (98\%), citric acid, itaconic acid ( $\geq 99 \%), 1,12$ dodecanedioic acid (99\%), and 2,2-dimethoxy-2-phenylacetophenone (99\%) were purchased from Sigma-Aldrich. Tetrahydrofuran (THF) was purchased from EMSURE. All of the chemicals were directly used as received.

\section{Synthesis of bio-based pre-polymer}

The bio-based pre-polymer was synthesized by catalyst-free polyesterification. First, 1,8-octanediol, citric acid, itaconic acid and dodecanedioic acid in certain molar ratio was melted in an oil bath at $160{ }^{\circ} \mathrm{C}$. As soon as all monomers melted, the temperature of the oil bath was lowered to $150{ }^{\circ} \mathrm{C}$. The photoinitiator, 2,2-dimethoxy-2phenylacetophenone (DMPA) was added in $20 \mathrm{wt} \%$ into reactant mixtures. After vigorously stirred for 6 hours, pre-polymer was obtained.

\section{Two-stage curing reactive method of bio-based polymer}

In first stage curing, the prepolymer was cured thermally at $100{ }^{\circ} \mathrm{C}$ for 24 hours. It was followed by second stage curing thru photo-induced free radical polymerization. The samples were exposed under ultraviolet irradiation $(365 \mathrm{~nm}, 6-10 \mathrm{~W})$. Time of exposure under UV irradiation was varied from 0 to $60 \mathrm{~min}$. Here, the obtained bio-based polymer was named as $\operatorname{PODC}_{x} \mathrm{I}_{\mathrm{y}} \mathrm{T}_{\mathrm{z}}$, where $\mathrm{x}$ is the molar ratio of citric 
acid, $\mathrm{y}$ is the molar ratio of itaconic acid, and $\mathrm{z}$ is the time of UV irradiation. Fig. 1 presents the schematic diagram for the synthesis of two-stage curing reactive bio-based polymer.

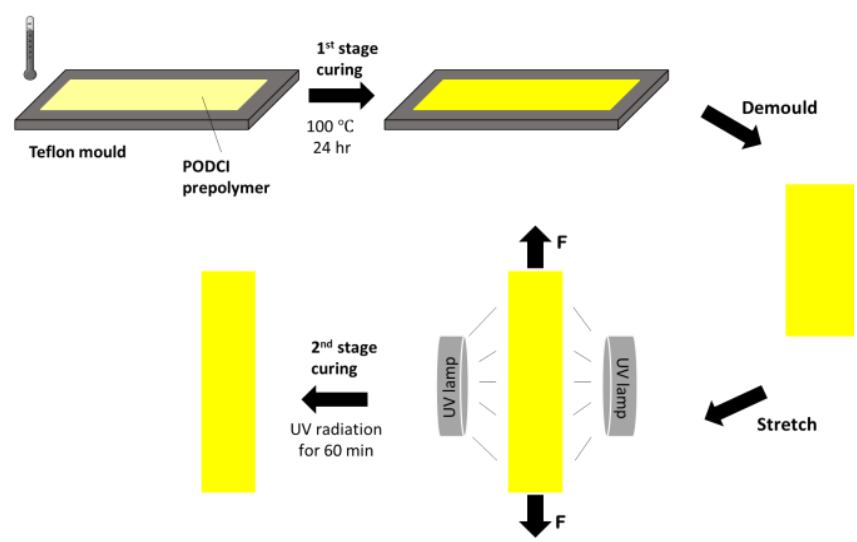

Fig. 1 Schematic diagram for the synthesis of a two-stage curing reactive process.

\section{Characterizations and testing}

The light transmission of PODCI was evaluated via ultravioletvisible light spectrometer (Shimadzu UV-3101PC, Japan). Fourier transform infrared spectra of PODCI was obtained from ATR-FTIR (NICOLET IS10-IR Spectrometer). Sol and gel content of PODCI was quantified by immersion in THF solvent via sol-gel analysis. The gel fraction $(\mathrm{G})$ and swelling ratio $(\mathrm{Q})$ were calculated according to Cardoso et al. (2011). The X-ray diffraction testing was conducted at ambient temperature on a Rigaku Dmax/III diffractometer using $\mathrm{Cu} \mathrm{Ko}$ radiation $(\lambda=1.54 \mathrm{~A})$ with accelerating voltage of $40 \mathrm{kV}$ and current of $30 \mathrm{~mA}$ respectively. The heating and cooling curve of PODCI was recorded under a nitrogen atmosphere by using a Differential scanning calorimetry (DSC) 820 (Mettler Toledo) within the temperature range of -50 to $100{ }^{\circ} \mathrm{C}$. In the first heating, the sample was heated from room temperature to $100{ }^{\circ} \mathrm{C}$ with a rate of $10^{\circ} \mathrm{C} \mathrm{min}-1$ and hold for $10 \mathrm{~min}$. It was followed by cooling to $-50{ }^{\circ} \mathrm{C}$ with a rate of $10{ }^{\circ} \mathrm{C} \mathrm{min}{ }^{-1}$ and then hold for another $10 \mathrm{~min}$. In second heating, the sample was reheated to $100{ }^{\circ} \mathrm{C}$ with the same heating rate as used in first heating. The shape memory property of PCI was quantified by determining the shape recovery from bending angle to original angle upon heating.

\section{RESULTS AND DISCUSSION}

\section{Light transmission study of PODC}

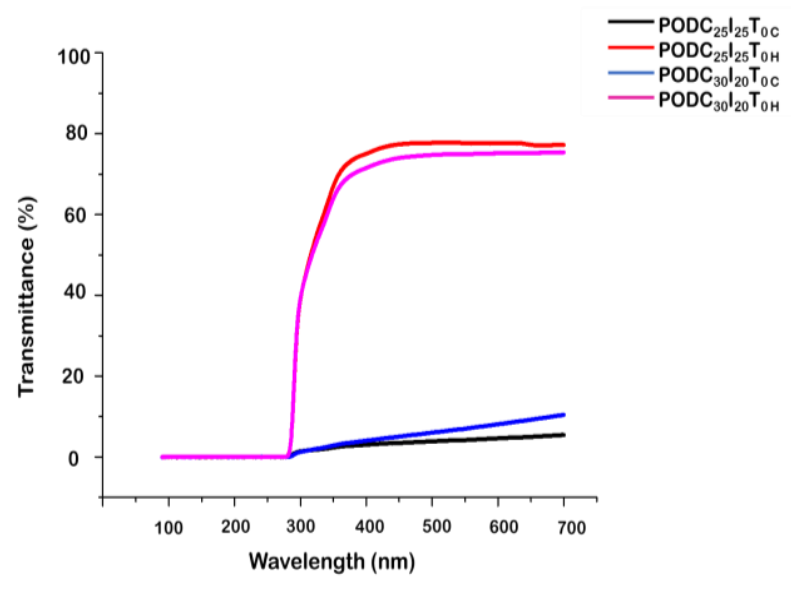

Fig. 2 UV light transmittance of PODCI.

Fig. 2 reveals the light transmittance of UV and visible spectral within 200-800 nm wavelength. At room condition, the light transmittance of all of the synthesized PODCI only achieved a maximum of $10 \%$. When it was heated above $50{ }^{\circ} \mathrm{C}$, the light transmittance increased to $77 \%$. At room temperature, the PODCI existed at semi-crystalline state, and it possessed opaque appearance. As soon as heated above the melting-transition temperature $\left(\mathrm{T}_{\mathrm{m}}\right)$, all of the crystalline structures were melted and it became transparent. Herein, second stage curing was conducted at a temperature higher than $\mathrm{Tm}$. We found that the PODCI cannot undergo UV-initiated free radical crosslink at a lower temperature.

\section{Chemical and structural properties of PODCI}

The gel fraction of one stage cured and two stages cured PODCI polymers were evaluated by quantifying the mass loss of sample after immersing into THF solvent. Fig. 3 shows the sol-gel analysis results of PODCI. Gel content refers to the crosslinked polymer network which is not soluble in THF. Due to network structure integrity, a prepolymer of PODCI able to undergo stage-wise curing. A prepolymer which soluble in THF was firstly thermally crosslinked and then followed by UV-initiated photocuring. From the result, the gel fraction of PODCI increased with increasing photocuring duration. It is well-known that crosslinked polymer cannot be remolded (reshaped). In this work, we presented that thermally crosslinked PODCI can be remolded easily upon completion of second stage curing. Photographs in Fig. 4 presents that a PODCI slab sample was successfully reshaped into helix dimension.

a

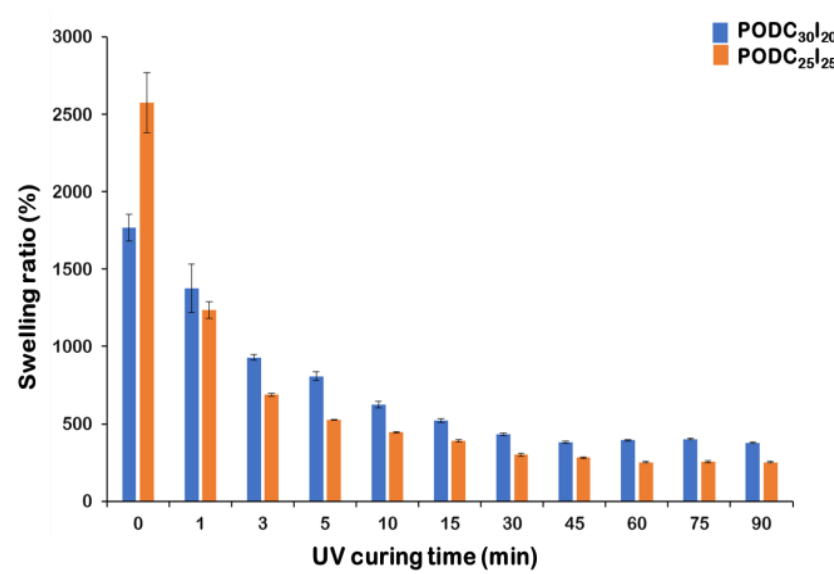

b

$-\mathrm{PODC}_{30} \mathrm{I}_{20}$ $\mathrm{PODC}_{25} \mathrm{I}_{25}$

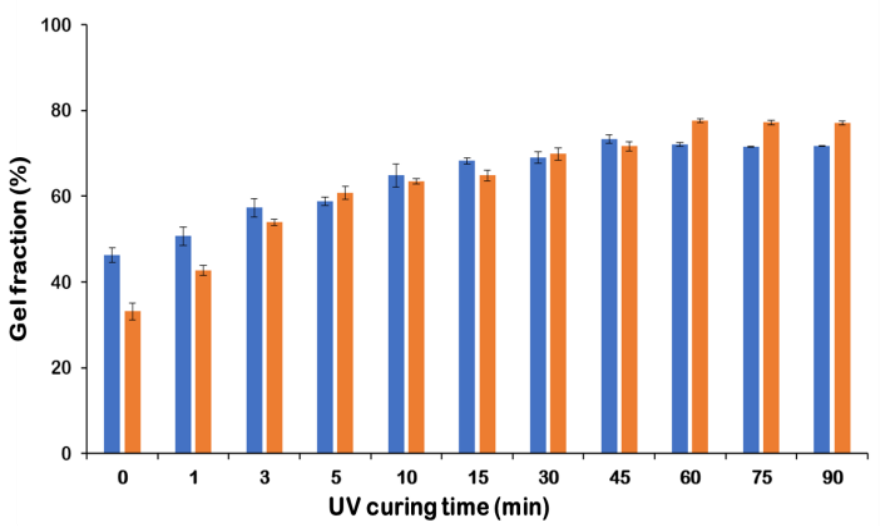

Fig. 3 (a) Swelling ratio and (b) Gel fraction with different photo-curing duration.

Fig. 4 presents a schematic diagram of crosslink formation during the stepwise-curing of PODCI. By introducing a UV light curable monomer, the IA, PODCI network can be further crosslinked upon application of UV irradiation. Swelling degree of PODCI was found decreased from $2570 \%$ to $250 \%$ after exposed under UV light for 60 min. PODCI with a greater content of IA was also found swelled lesser. As an alternative, the swelling degree is also an indication for crosslinking density which higher swelling degree indicates lower crosslinking density. 

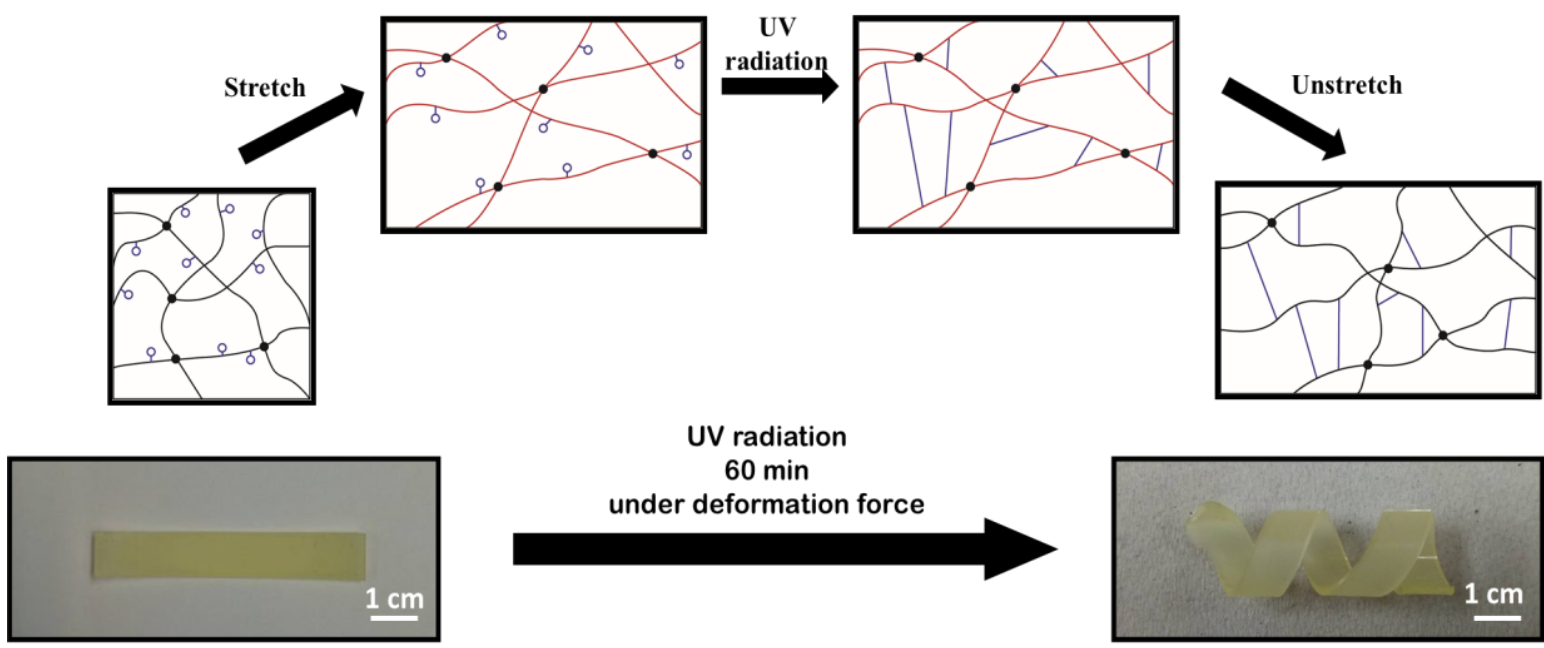

After $1^{\text {st }}$ curing stage

Relax state

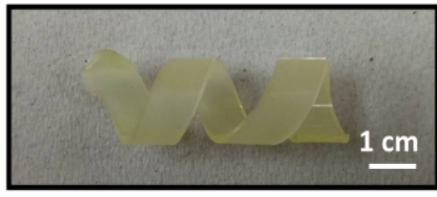

$\mathrm{O}^{-}=\mathrm{CH}_{2}$ bond

UV crosslinker

Stretching state

After $2^{\text {nd }}$ curing stage

Thermal crosslinker

Fig. 4 Schematic diagram of crosslinks formation and photo for reshaping of PODCl upon second curing process.

Fig. 5 shows FTIR spectra of PODCI. The transmission peak at $1370-1470 \mathrm{~cm}^{-1}$ is corresponding to $=\mathrm{C}-\mathrm{H}$ stretching from branches due to the presence of IA. The transmission peaks at $1735-1750 \mathrm{~cm}^{-1}$ attributed to the stretching of $\mathrm{C}=\mathrm{O}$ (ester bond) within the polymer network. Upon UV irradiation exposure, the carbon-carbon double bond $(=\mathrm{C}-\mathrm{H})$ of IA was transformed into alkyl group $\left(-\mathrm{CH}_{3}\right)$. It was clearly shown that peak intensity due to $=\mathrm{C}-\mathrm{H}$ double bond (absorption band at $1375 \mathrm{~cm}^{-1}$ ) was decreased while the band at $2930 \mathrm{~cm}^{-1}$ corresponding to the vibration of the alkyl group $\left(-\mathrm{CH}_{3}\right)$ was increased. While the transmission peak at $1370-1470 \mathrm{~cm}^{-1}$ still showed at FTIR spectra because the uncrosslinked $=\mathrm{C}-\mathrm{H}$ still remains at the branches of PODCI networks.
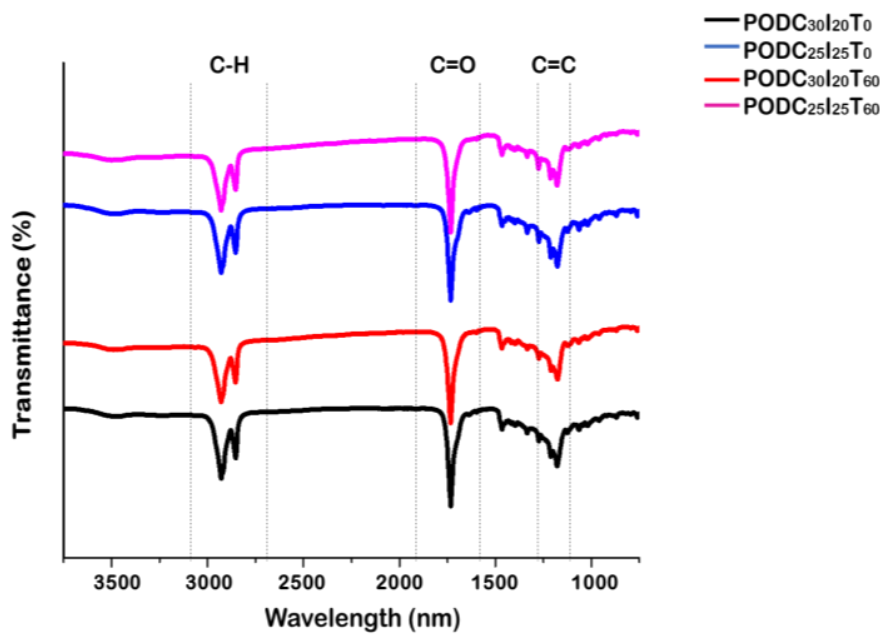

Fig. 5 FTIR spectra of PODCI.

Fig. 6 presents X-ray diffraction (XRD) pattern of the PODCI with a different stoichiometric ratio of CA to IA and different photocuring time. From the diffraction pattern of PODC30I20T0, the diffraction peaks around 21.3 and 23.9 are significantly high. After second stage curing, the intensity of these diffraction peaks remarkably decreased. The degree of polymer crystallinity $\left(\mathrm{X}_{\mathrm{c}}\right)$ was calculated using Eq. (1).

$$
X_{c}=\sum_{i-1}^{i=n} A_{c i} / A_{t}
$$

where $A_{c i}$ is the under each crystalline peak with index $I$ and $A_{t}$ is the total area under the diffractogram (Lopez-Rubio et al., 2008). The Xc of PODCI were recorded in Table 1. The Xc of PODCI was found decreased after further curing under UV light. The increment in polymer crosslinking inhibits polymer crystallization.
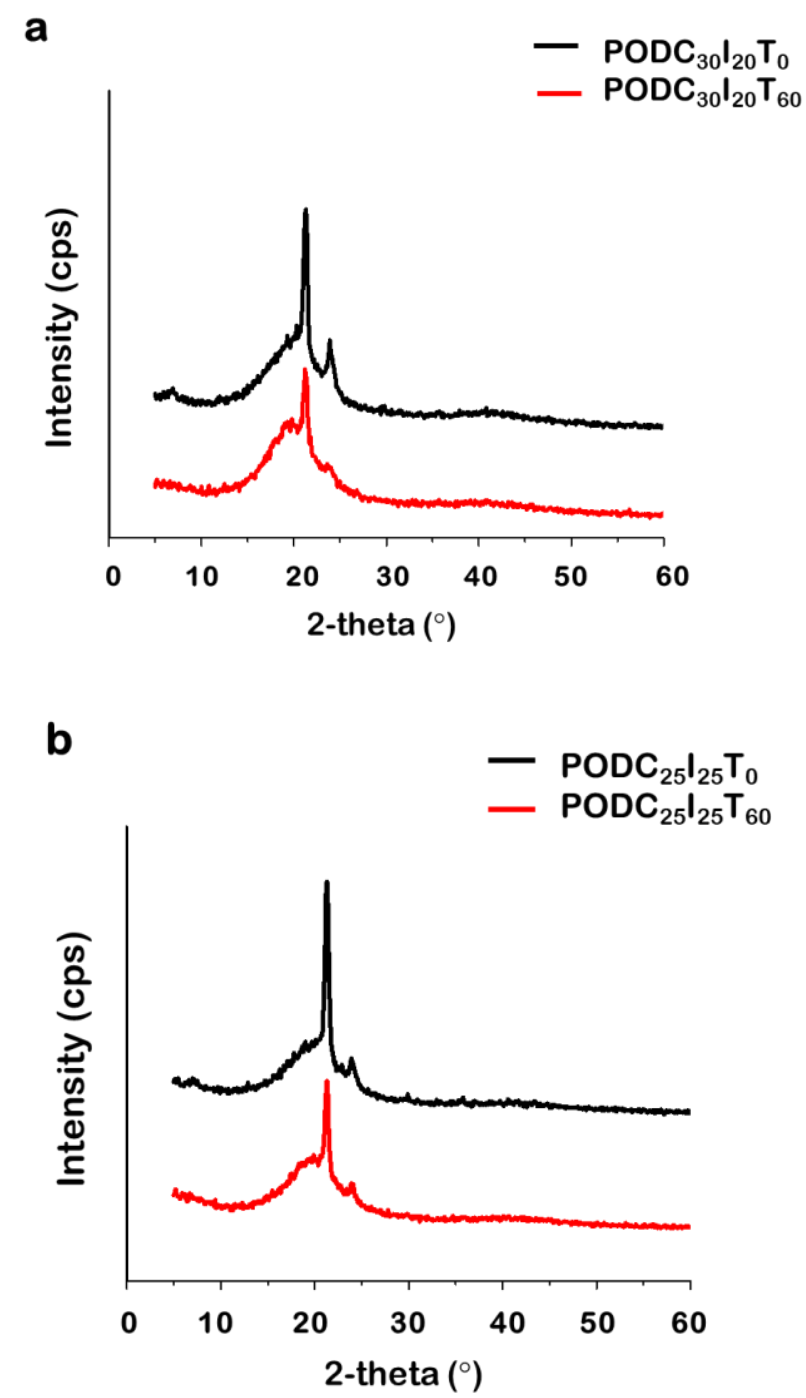

Fig. 6 XRD spectra for (a) PODC30I20 and (b) PODC25I25 with two different photo-curing time. 
Table 1 Thermal data and degree of polymer crystallinity of PODCI.

\begin{tabular}{|c|c|c|c|}
\hline Formulation & $\mathrm{Tc}_{\mathrm{c}}{ }^{\mathrm{a}}\left({ }^{\circ} \mathrm{C}\right)$ & $\mathrm{Tm}^{\mathrm{b}}\left({ }^{\circ} \mathrm{C}\right)$ & $X_{c^{c}}(\%)$ \\
\hline $\mathrm{PODC}_{30} \mathrm{I}_{20} \mathrm{~T}_{0}$ & 1.5 & 40.4 & 17.7 \\
\hline $\mathrm{PODC}_{30} \mathrm{I}_{20} \mathrm{~T}_{60}$ & -17.8 & 28.9 & 9.6 \\
\hline $\mathrm{PODC}_{25 \mathrm{I}_{25} \mathrm{~T}_{0}}$ & 9.2 & 44.2 & 20.5 \\
\hline $\mathrm{PODC}_{25} \mathrm{I}_{25} \mathrm{~T}_{60}$ & 9.1 & 40.2 & 10.4 \\
\hline
\end{tabular}

a Crystallization transition temperature determined by a first cooling curve of DSC.

b Melting transition temperature determined by a second heating curve of DSC.

c Polymer crystallinity of PODCl calculated by Eq. (1).

\section{Thermal properties of PODCI}

Fig. 8 depicts the DSC thermograms curve of PODCI during cooling and second heating process. The thermal properties of PODCI were displayed in Table 1. From thermograms, it was found that the crystallization transition temperature $\left(\mathrm{T}_{\mathrm{c}}\right)$ of PODCI is below $9.2{ }^{\circ} \mathrm{C}$ When IA content is increased, leading to the decreased possibility of branch forming by CA after first stage curing. Therefore, the melting transition temperature of $\mathrm{PODC}_{30} \mathrm{I}_{20}$ is lower than it's of $\mathrm{PODC}_{25} \mathrm{I}_{25}$. Melting transition temperature of PCI decreased after second stage curing, as the crystalline of PCI decreased with increasing crosslinking formed. The DSC results indicate that PCI exhibits semi-crystalline state at room condition.
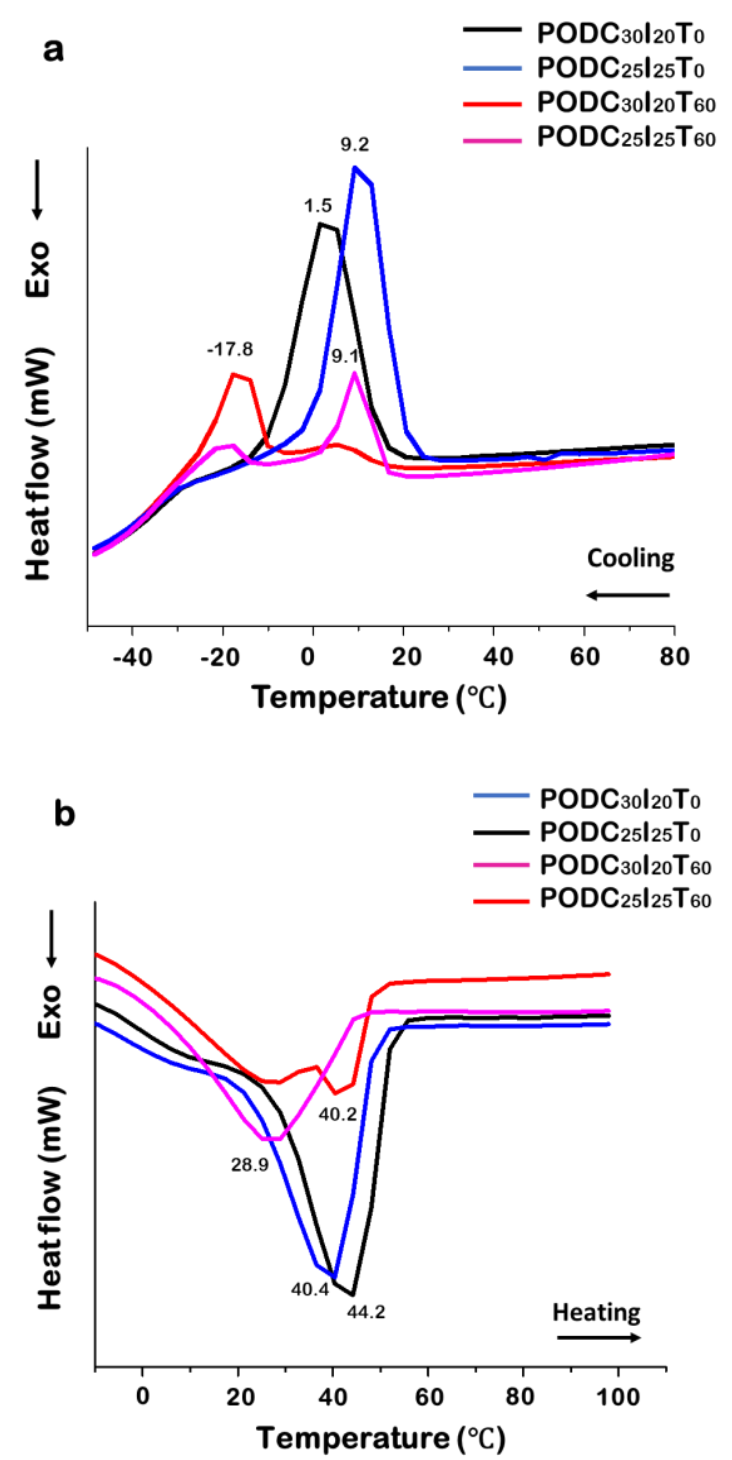

Fig. 8 DSC curve for (a) cooling curve and (b) second heating curve of PODCI.

\section{Shape memory effect of PODCI}

PODCI is a shape memory polymer as it can be fixed in a temporary shape and only regain its permanent shape upon heating. Fig. 9a presents the relationship between shape recovery percentage and change in temperature upon heating and Fig. 9b displays the images of PODCI in water bath upon heating. The shape recovery percentage $\left(R_{r}\right)$ was calculated using Eq. (2).

$$
R_{r}=\left(180^{\circ}-A_{d}\right) / A_{o} \times 100 \%
$$

where $A_{d}$ is deformed angle and $A_{o}$ is original angle. With the increasing temperature of the water bath, the temporary shape of PODCI started to recover at $23{ }^{\circ} \mathrm{C}$, and it fully recovered to its permanent shape at a temperature of $42^{\circ} \mathrm{C}$. As well as the higher deformation recovery temperature of PODCI is $36^{\circ} \mathrm{C}$. PODCI possessed an excellent shape memory behavior with shape recovery temperature of $36^{\circ} \mathrm{C}$. Therefore, PODCI can act as an actuator or drug capsules within the human body.

a

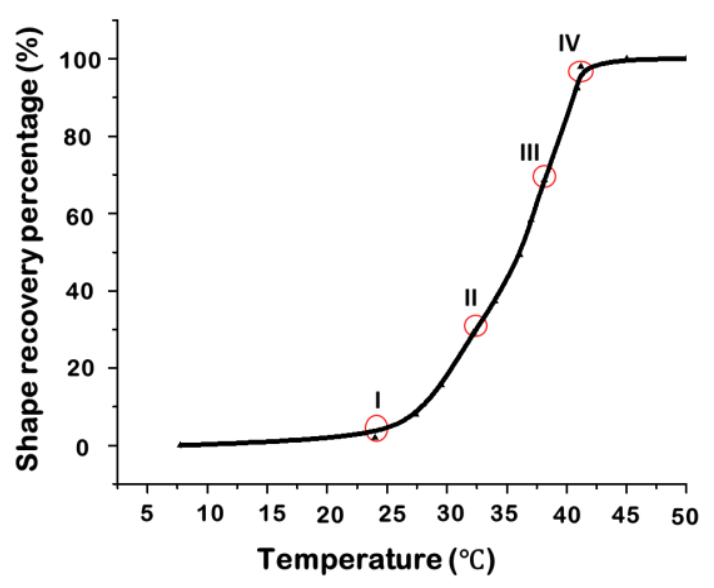

b

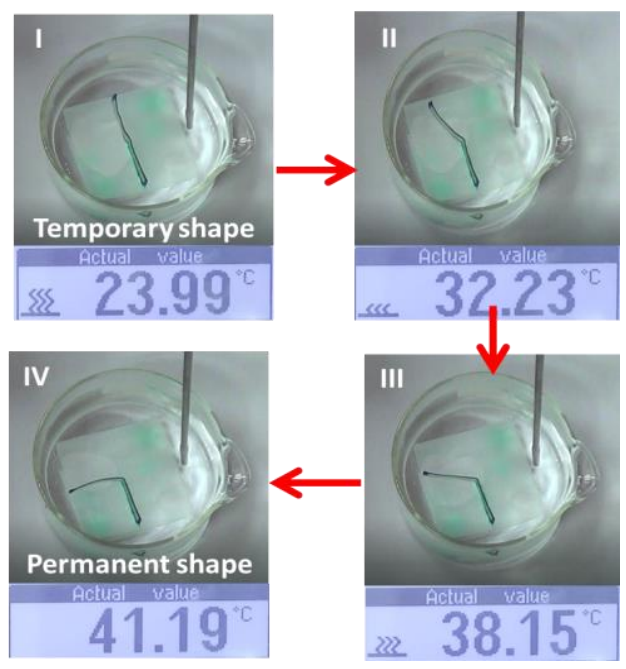

Fig. 9 (a) Relationship between shape recovery percentage of PODCl and temperature (b) Image of angle deformation change of PODCI with temperature.

\section{CONCLUSION}

A two-stage cured bio-based polymer was successfully synthesized. The shape of the polymer can be tailored after the second stage by free-radical photopolymerization. PODCI possessed different chemical, structural, and thermal properties between one stage and two stages curing reactive process. Two-stage cured PODCI possessed an excellent shape memory property. 


\section{ACKNOWLEDGEMENT}

This work was financially supported by the Universiti Teknologi Malaysia under the Research University Grant and Ministry of Higher Education Malaysia.

\section{REFERENCES}

Cardoso, E. C. L., Seagllust, S. R., Moraes, G. F., Ono, L. S., Parra, D. F., \& Lugao, A. B. (2011, October 24-28). Density crosslink study of gammairradiated LDPE predicted by gel-fraction, swelling and glass transition temperature characterization. Paper presented at International NuclearAtlantic Conference, Brazil.

Liu, Y., Boyles, J. K., Genzer, J., and Dickey, M. D. (2011). Self-folding of polymer sheets using local light absorption. Soft Matter, 8 (6): 1703-2044.

Lopez-Rubio, A., Flanagan, B. M., Gilbert, E. P., and Gidley, M. J. (2008). A novel approach for calculating starch crystallinity and its correlation with double helix content: A combined XRD and NMR study. Biopolymer, 89(9): 761-768.

Meng, Y., Jiang, J., Anthamatten, M. (2014). Shape actuation via internal stress induced crystallization of dual-cure networks. ACS Macro Letters, 2015(4): 115-118.

Miao, S., Nicholas, C., Wang, Pi., Liu, Y., Su, Z., and Zhang, S. (2013). Soybean oil based polyurethane networks: Shape-memory effects and surface morphologies. Journal of the American Oil Chemists' Society, 90: 14151421

Serrano, M. C., Carbajal, L., Ammer, G. A. (2011). Novel biodegradable shape memory elastomers with drug-releasing capabilities. Advanced Materials, 2011 (23): 2211-2215

Xie, T. (2011). Recent advances in polymer shape memory. Polymer, 52 (2011) 4985-5000.

Yakacki, C. M., Saed, M., Nair, D. P., Gong, T., Reed, S. M., and Bowman, C.N (2015). Tailorable and programmable liquid-crystalline elastomers using a two-stage thiol acrylate reaction. The Royal Society of Chemistry, 2015(5): 18997-19001.

Yang, J., Webb, A. R. (2004). Novel citric acid-based biodegradable elastomers for tissues engineering. Advanced Materials, 16 (60): 511-516. 